\title{
THERAPEUTIC EFFICACY OF INTRAPERITONEALLY INJECTED CURCUMIN ON EXPERIMENTALLY INDUCED HAMSTER BUCCAL POUCH CARCINOMA
}

\author{
Emad Mohammed Alqalshy*, Ahmed Al Mohamady *
}

\begin{abstract}
Objective: The aim of the present study was directed to investigate the therapeutic efficacy of intraperitoneally injected curcumin as anticarcinogenic treatment modality on 7,12-dimethylbenz[a]anthracene (DMBA) induced hamster buccal pouch(s) (HBP(s)) carcinoma. Subjects and methods: Materials and Methods: Thirty Syrian male hamsters, five weeks old, weighing 90$130 \mathrm{~g}$ were divided into three group(s) $(\mathrm{G}(\mathrm{s})$ ): A, B, and C. G-A (negative control): HBP mucosa of 10 animals were painted with liquid paraffin alone three times a week for 14 weeks. G-B (positive control): 10 animals were painted with $0.5 \%$ DMBA in paraffin oil 3 times a week for 14 weeks. Then animals were left untreated for 6 weeks after DMBA painting. G-C (curcumin treated group): 10 animals were injected intraperitoneally with curcumin $80 \mathrm{mg} / \mathrm{kg}$ body weight every day for 6 weeks following DMBA-treatment. The assessment was based on the gross observation, histological and immunohistochemical examination utilizing anti-apoptotic Bcl-2 antibody. Results: Gross observation revealed variation in reduction of the tumor size in the treated group (G-C) compared to that observed in group G-B. Histopathological findings revealed variations between $\mathrm{G}-\mathrm{B}$ and $\mathrm{G}-\mathrm{C}$ groups. Immunohistochemical results revealed that Bcl-2 expression has a variability in the area \% throughout the groups used. Groups G-A, G-B, and G-C were $7.78 \%, 75.51 \%$, and $41.65 \%$ respectively. Conclusion: The therapeutic effect of intraperitoneally injected curcumin resulted in promising outcome in regression of DMBA induced HBP carcinoma.
\end{abstract}

KEYWORDS: HBP carcinoma, DMBA, curcumin.

\section{INTRODUCTION}

Oral cancer refers to a subgroup of head and neck malignancies that located within the top 10 ranking prevalence of cancers globally ${ }^{(1)}$. The yearly incidence is higher around the world, which is more than 300.000 diagnosed cases, and the annual mortality is about 145,000 deaths $^{(2)}$. Oral squamous cell carcinoma (OSCC) constitute over $90 \%$ of oral cancer $^{(3)}$. It has been found that 7,12-dimethylbenz(a) anthracene (DMBA) induced hamster buccal pouch (HBP) carcinogenesis closely emulate the human
OSCC on biochemical, morphological and histological aspects as well as at molecular level ${ }^{(4)}$. The alternative natural compounds had been shown to promote the effects of the chemotherapeutic drugs, reduce obstacles of chemotherapy, and increase the sensitivity of cancer cells ${ }^{(5)}$.

One of these alternatives is curcumin, a natural component that is derived from turmeric ${ }^{(6)}$.Curcumin (1,7-bis(4-hydroxy-3-methoxyphenyl)-1-6-heptadiene-3,5dione,cm) or diferuloylmethane, is a polyphenol that can be found in Curcuma species,

* Lecturer, Oral and Dental Pathology Department, Faculty of Dental Medicine, (Boys-Cairo), Al-Azhar University, Egypt

•Corresponding author: emadalqalshy.209@azhar.edu.eg 
such as turmeric (Curcuma longa) and javanese ginger (Curcuma xanthorrhiza). Curcumin is composed of $77 \%$ bis- $\alpha, \beta$-unsaturated $\beta$-diketone or diferuloylmethane or curcumin, $18 \%$ demethoxycurcumin, and 5\% bisdemethoxycurcumin. Curcumin has been known for its various therapeutic potency, such as antiseptic, analgesic, anti-inflammation, antioxidant, antimalarial, antivirus, and antitumor or anticarcinogenic ${ }^{(7)}$. It has been shown that curcumin alone or in combination with other drugs increase and promote cell death in a wide variety of tumor cells, including HNSCC ${ }^{(8)}$.

One of the most forced reasons for continued interest in scouting the cancer chemopreventive and chemotherapeutic uses of curcumin has been curcumin's ability to influence a wide range of molecular targets within cells ${ }^{\left({ }^{9}\right)}$. Indeed, curcumin has been shown to induce apoptosis via the up-regulation of pro-apoptotic proteins and down-regulation of anti-apoptotic proteins of the Bcl-2 family in cancer cells which results in cell death ${ }^{(10)}$. Hence, the main target of the present study was to assess the therapeutic efficacy of curcumin as anticancer treatment modality in DMBA induced HBP carcinoma. The assessment was based on the gross observation, histological tumor tissue changes and immunohistochemical examination using anti-apoptotic Bcl-2 antibody.

\section{MATERIALS AND METHODS}

Thirty Syrian male hamsters five weeks old, weighing 90-130g were obtained from the animal house, Cairo University (Cairo, Egypt). The experimental animals were housed in standard cages under controlled environmental conditions of humidity $(30-40 \%)$, temperature $\left(20 \pm 2^{\circ} \mathrm{C}\right)$, and light (12h light/12-h dark). All experimental animals were supplied with standard diet and water ad libitum. 7,12 DMBA $(0.5 \%)$ was obtained from Sigma-Aldrich company, dissolved in paraffin oil and used thrice a week for 14 weeks via Painting of right HBP using a number 4 camel's hair brush ${ }^{(11,12)}$. Curcumin was obtained from Sigma-Aldrich Company, dissolved in dimethyl sulfoxide (DMSO), and used via intraperitoneal injection as $80 \mathrm{mg} / \mathrm{kg}$ body weight, every day, for 6 weeks ${ }^{(13)}$.

Experimental design: Thirty Syrian male hamsters were divided into three G(s). G-A (negative control): HBP mucosa of 10 animals were painted with liquid paraffin alone three times a week for 14 weeks. G-B: 10 right HBP animals were painted with $0.5 \%$ DMBA in paraffin oil using 3 times a week for 14 weeks. Then animals were left untreated for 6 weeks after DMBA painting. G-C (curcumin treated group): Right HBP of 10 animals were intraperitoneally injected with curcumin $80 \mathrm{mg} /$ $\mathrm{kg}$ body weight every day for 6 weeks following DMBA-treatment ${ }^{(14)}$.

Investigations: After termination of the experiment, all animals were euthanized, the cheek pouches were everted, and the diameter of each tumour was measured with a Vernier caliper. The tumour volume was calculated by the formula, $\mathrm{Vmm}^{3}=$ $(4 / 3) \pi((\mathrm{D} 1 / 2)(\mathrm{D} 2 / 2)(\mathrm{D} 3 / 2))$, where D1, D2 and D3 are the three diameters $(\mathrm{mm})$ of the tumour ${ }^{(4)}$. Then, the hamster's head were separated, the cheek pouches were excised and fixed in $10 \%$ neutral buffered formalin, routinely processed and embedded in paraffin blocks for preparation in order to be examined histologically and immunohistochemically.

For histopathological examination: The fixed specimens were dehydrated in an ascending ethanol series, embedded in paraffin wax to form paraffin blocks. Tissue sections of $4 \mu \mathrm{m}$ thickness on rotary microtome were cut, mounted on glass slides, processed, and stained with H\&E for light microscopic examination.

For immunohistochemical examination: Other tissue sections were cut at $5 \mu \mathrm{m}$ thickness for the 
application of standard labeled streptavidin- biotin method to illustrate the expression of mouse monoclonal Bcl-2 antibody. The Paraffin embedded tissue sections were dewaxed and rehydrated through graded ethanol to distilled water. Endogenous peroxidase was blocked by incubation with $3 \%$ $\mathrm{H} 2 \mathrm{O} 2$ in methanol for 10 minutes. The antigen retrieval was achieved by adding citrate buffer solution ( $\mathrm{pH}$ 6.0) and keeping in microwave for 10 min, followed by washing with Tris-buffered saline ( $\mathrm{pH}$ 7.6). The tissue sections were then incubated with the proteinaceous blocking reagent power BlockTM for 15 min at room temperature to block non-specific binding, and further with the respective primary antibody $\mathrm{Bcl}-2$ at $4{ }^{\circ} \mathrm{C}$. The bound primary antibody was detected by incubation with their corresponding secondary antibodies, conjugated with horseradish peroxidase for $30 \mathrm{~min}$ at room temperature. After rinsing with Tris-buffered saline, the antigen-antibody complex was detected using 3,3-diaminobenzidine (Sigma, USA), the substrate of horseradish peroxidase. When acceptable color intensity reached, the slides were washed, counter stained with Mayer's haematoxylin and covered.

The immunostained sections were examined using light microscope to assess the prevalence of positive cases and the localization of immunostaining within the tissues. In addition, image analysis computer system was used to assess area percentage of positive cells of the immunostaining. This was done at the Oral and Dental Pathology Department - Faculty of Dental Medicine - Boys- Cairo - AlAzhar University. The degree of positive staining for antibody was evaluated by a well-established quantitative scoring on a scale rang from negative to strong positive staining as follow: Strong staining (more than 50\% stained), moderate staining (between 25 and 50\% stained), weak staining (between 5 and $25 \%$ stained), and negative (less than $5 \%$ stained) ${ }^{(15)}$.

\section{RESULTS}

The gross observation results of HBP mucosa of G-A was light pink in color with smooth surface texture with no observable abnormalities (Fig.1:A). In G-B, HBP mucosa revealed various changes including variable sized nodular elevations and exophytic papillary tumor masses. Vernier caliper revealed that the mean tumor volume of tumorbearing animals was $845.7 \mathrm{~mm}^{3}$ with $100 \%$ tumor formation. Furthermore, eroded and ulcerative areas were seen throughout the tumor growth, with spontaneous profound bleeding (Fig.1:B). In G-C, HBP mucosa showed that intraperitoneal injection of curcumin $80 \mathrm{mg} / \mathrm{kg}$ body weight every day for 6 weeks following DMBA-treatment revealed different sizes of exophytic tumor masses with marked decrease in tumor volume. Vernier caliper revealed that the mean tumor volume of tumorbearing animals was $201.9 \mathrm{~mm}^{3}$ (Fig.1:C).

Histopathological and immunohistochemical results: The tissue sections of HBP mucosa of experimental groups showed variable results in regard to the histopathological results and immunohistochemical results. In G-A, histological sections, using H\&E stain, revealed normal HBP mucosa, with thin stratified squamous epithelium without rete pegs with submucosal dense fibrous connective tissue and layer of longitudinal striated muscle fibers (Fig.2:A). The immunohistochemical staining using Bcl-2 antibody exhibited weak positive (7.78\%) cytoplasmic expression of basal and supra-basal layers and negative in the remaining epithelial cell layers (Fig.2:B). In G-B, histological sections, using $\mathrm{H} \& \mathrm{E}$ stain, showed moderately to poorly differentiated SCC with deeply invading islands of tumor cells into the underlying connective tissue. The surface epithelium exhibited severe epithelial dysplasia (Fig.2:C). The immunohistochemical staining using Bcl-2 antibody exhibited strong positive (75.51\%) cytoplasmic expression throughout the epithelial layers and invading tumor cells (Fig.2:D). In G-C, histological sections, using H\&E stain, 
revealed well differentiated SCC with superficial invasion of tumor islands and keratin pearls which was juxta-epithelial and not extended to the deeper connective tissue with increased amount of keratin formation. The surface epithelium showed severe epithelial dysplasia with hyperkeratosis (Fig.2:E). The immunohistochemical staining using Bcl-2 antibody exhibited moderate positive $(41.65 \%)$ cytoplasmic expression throughout the epithelial layers and invading tumor cells (Fig.2:F).

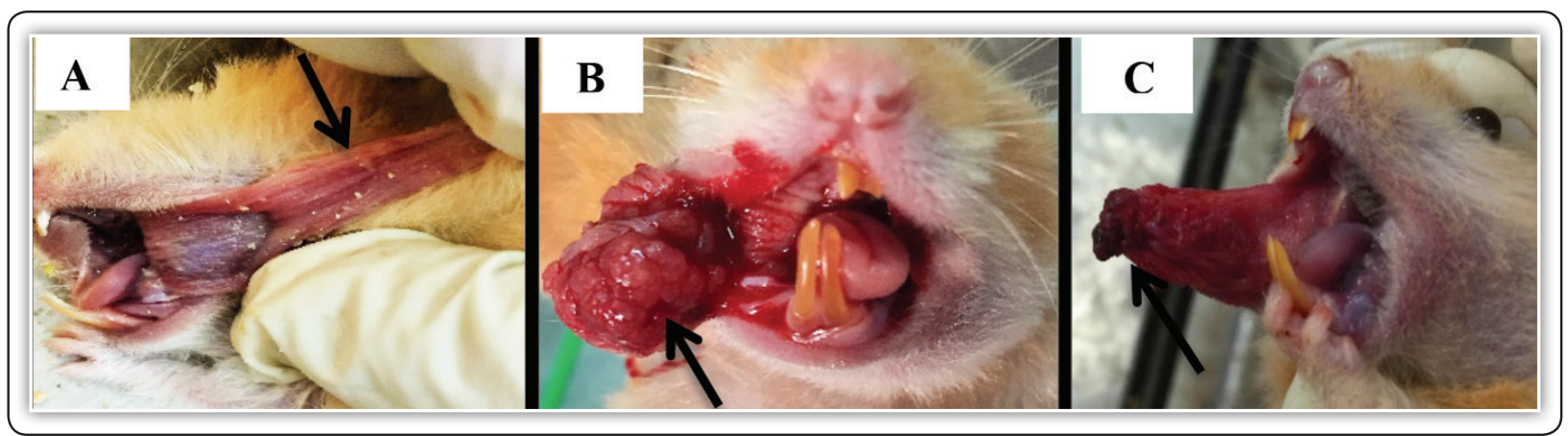

FIG (1) (A): HBP mucosa of G-A was pink in color with no observable abnormalities (arrow). Fig.1(B): HBP mucosa of G-B1 showed variable sized nodular elevations and exophytic papillary tumor masses with bleeding and ulcerations (arrow). Fig.1(C): HBP mucosa of G-B3 showed small exophytic tumor masses with marked decrease in distribution and size of tumor masses, and absence of bleeding and ulceration (arrow).

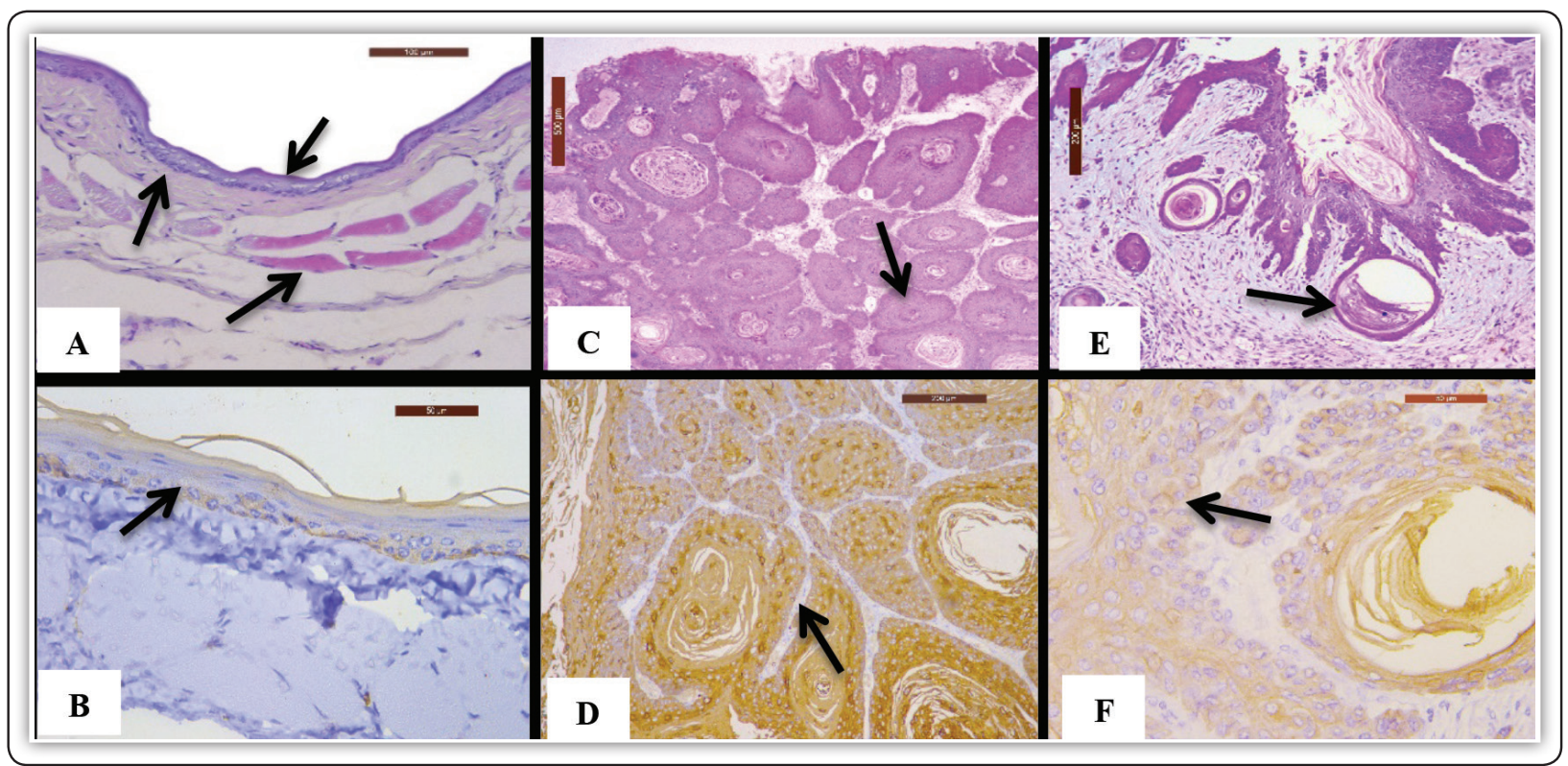

FIG (2) (A): Photomicrograph of G-A showed normal HBP mucosa, composed of thin stratified squamous epithelium without rete ridges with submucosal dense fibrous connective tissue and layer of longitudinal striated muscle fibers (arrows). Fig.2(B): Bcl-2 expression in G-A showed weak positive cytoplasmic expression of basal and supra-basal layers (arrow). Fig.2(C): Photomicrograph of G-B showed well to moderately differentiated SCC with deeply invading islands of tumor cells into the underlying connective tissue (arrow). Fig.2(D): Bcl-2 expression in G-B showed strong positive cytoplasmic expression throughout the epithelial layers and invading tumor cells (arrow). Fig.2(E): Photomicrograph of G-C showed well differentiated SCC which with juxta-epithelial and superficial invasion of tumor islands and keratin pearls with increased amount of keratin formation (arrow). Fig.2 (F): Bcl-2 expression in G-C showed moderate positive cytoplasmic expression throughout the epithelial layers and invading tumor cells (arrow). 
Statistical analysis results of Bcl-2 expression were obtained by comparing its area $\%$ in the groups used. Statistical analysis results revealed that G-A has recorded the lowest mean area \% (7.78\%), while G-B had the highest mean area \% (75.51\%), G-C showed statistically lower mean value than G-B (41.65\%) (Fig.3).

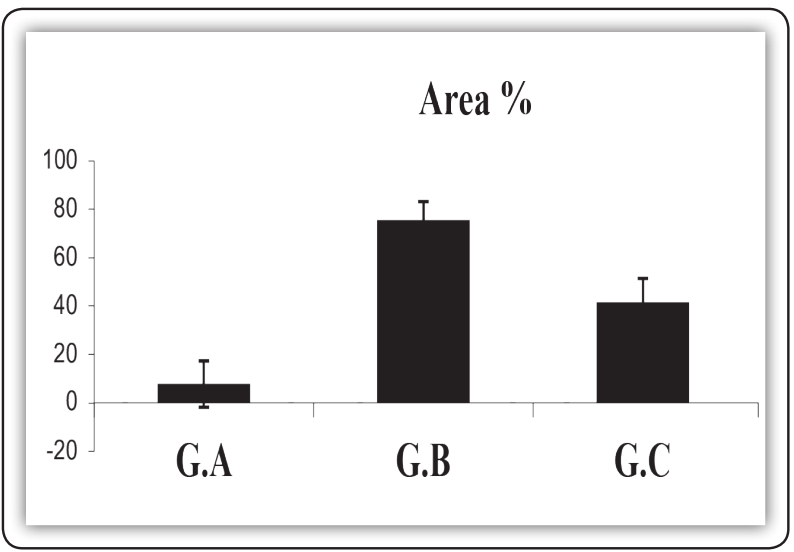

FIG (3) Bar chart representing mean area \% results among different groups which G-A has recorded the lowest mean area \% (7.78\%), G-B had the highest mean area $\%(75.51 \%)$, and G-C showed statistically lower mean value than G-B $(41.65 \%)$.

\section{DISCUSSION}

Oral cancer, one of the most damaging types of cancer and in spite of the notable advancement in oral cancer treatment strategy; it remains as a major cause of morbidity in human populations. The use of hamster cheek pouch system of oral carcinogenesis model is valuable for deeper understanding of cancer biology, prevention and treatment. In the present study, the gross observation findings in G-A showed no observable abnormalities of the HBP. After euthanization and head separation, the buccal pouches length was about $4-5 \mathrm{~cm}$ for all hamsters with normal histological structures. Other studies reported the same findings $(16,17)$. Immunohistochemical staining of normal hamster mucosal tissue showed that weak positive $(7.78 \%) \mathrm{Bcl}-2$ localization was confined to the basal and supra-basal layers and negative in the remaining epithelial cell layers.
This result is in accordance with that of other investigators ${ }^{(18,19)}$. This observation might be relate to that up regulation of $\mathrm{Bcl}-2$ in basal and supra-basal cells serve to maintain the keratinocyte stem cells from apoptosis, and that negative Bcl-2 expression in the remaining epithelial layers collateral with terminal cell differentiation ${ }^{(20)}$.

In the present study, the gross observation of HBP in G-B showed variable sized nodules and exophytic papillary tumor masses with mean tumor volume of tumor-bearing animals were $845.7 \mathrm{~mm}^{3}$ and $100 \%$ tumor formation. Furthermore, eroded and ulcerative areas were seen throughout the tumor growth, with spontaneous bleeding. These results are almost the same with that shown by other investigators ${ }^{(21-24)}$. Histopathological results of G-B showed moderately to poorly differentiated SCC with deeply invading tumor islands into the underlying connective tissue. The surface epithelium showed hyperkeratinization, and elongated dropshaped rete pegs with areas of variable degrees of dysplasia. These results are concomitant with those of other investigators ${ }^{24,25)}$. These observations might be interpreted as DMBA induced over production of reactive oxygen species, chronic inflammation, oxidative modification of DNA bases, impairment in antioxidant defense system, defect in the activities of detoxification cascade and deregulated expression pattern of molecular markers are involved in the promotion and progression of oral carcinogenesis ${ }^{(17)}$.

The Bcl-2 immunohistochemical results of G-B showed strong positive (75.51\%) cytoplasmic expression throughout the epithelial layers and invading tumor cells. These results are in line with those of other investigators ${ }^{(17,26,27)}$. This suggests that overexpression of $\mathrm{Bcl}-2$ indirectly inhibits the process of apoptosis and thus plays a role not only in the onset of tumorigenesis, but also influences the progression of the disease because it increases the survival rate of neoplastic cells, allowing new genetic mutations to occur and making them higher resistant to treatment ${ }^{(27)}$. 
In the present work, the gross observation of HBP in G-C showed improvement in general health of animals. An exophytic tumor mass showing decrease in tumor volume which was statistically significant lower than G-B with mean tumor volume of tumor-bearing animals was $201.9 \mathrm{~mm}^{3}$. These results are in agreement with other studies which report the growth suppressive effects of curcumin on HNSCC ${ }^{(6,28-30)}$. Guha et al. reported that growth suppression effect was done by the effect of curcumin on the nuclear factor kB (NF-kB) signaling pathway. Curcumin caused a decrease in the expression of NF-kB and, in addition, inhibited its nuclear localization. The activity of curcumin on the NF-kB in SCC is due to inhibition of IkB kinase, resulting in NF-kB sequestration in the cytoplasm. As a result, curcumin has been shown to suppress the expression of a variety of NF-kB regulated gene products concerned in carcinogenesis and tumor growth ${ }^{(31)}$.

Histopathological results of G-C revealed invasion of well differentiated SCC which was juxta-epithelial and not extended to the underlying deeper connective tissue. These results were in line with Hung et al. in which they reported that curcumin evidently reduced cell number migrating through the connective tissue. The observed effect of curcumin on cell invasion may be partially assisted by decreasing activity of extracellular matrix proteases which play an important roles in local invasion. The Bcl-2 immunohistochemical results of G-C showed moderate positive cytoplasmic expression throughout the epithelial layers and invading tumor cells ${ }^{(32)}$.

This result is in agreement with Ravindran et al. and Nor Isnida Ismail et al. who postulated that curcumin induces downregulation of anti-apoptotic proteins and up regulation of pro-apoptotic proteins that lead to a loss of the mitochondrial membrane potential, opening of the transition pore, releasing cytochrome c, activating caspase-9 and caspase-3, and ultimately DNA fragmentation and apoptosis.
Furthermore, curcumin was found to induce apoptosis in cancer cells by continuous increase in $\mathrm{Ca} 2+$ through the down-regulation of $\mathrm{Bcl}-2$ protein. The disruption of mitochondrial outer membrane guide to the release of cytochrome $\mathrm{C}$ and subsequently undergone apoptosis ${ }^{(33,34)}$.

In conclusion, the effect of intraperitoneally injected curcumin could be a favorable treatment of that DMBA induced HBP carcinoma model not only in the gross observation results but also in histopathological and immunohistochemical results. This was enhanced by inducing apoptosis via downregulation of the $\mathrm{Bcl}-2$ protein.

\section{REFERENCES}

1. Rivera, C., Essentials of oral cancer. International journal of clinical and experimental pathology, 2015. 8(9): p. 11884.

2. Ferlay, J., et al., Cancer incidence and mortality worldwide: sources, methods and major patterns in GLOBOCAN 2012. International journal of cancer, 2015. 136(5): p. E359-E386.

3. Jemal, A., et al., Cancer occurrence. Cancer Epidemiology, 2009: p. 3-29.

4. Silvan, S. and S. Manoharan, Apigenin prevents deregulation in the expression pattern of cell-proliferative, apoptotic, inflammatory and angiogenic markers during 7, 12-dimethylbenz (a) anthracene-induced hamster buccal pouch carcinogenesis. Archives of oral biology, 2013. 58(1): p. 94-101.

5. Kienle, G.S., et al., Viscum album L. extracts in breast and gynaecological cancers: a systematic review of clinical and preclinical research. Journal of experimental \& clinical cancer research, 2009. 28(1): p. 1.

6. Wilken, R., et al., Curcumin: A review of anti-cancer properties and therapeutic activity in head and neck squamous cell carcinoma. Molecular cancer, 2011. 10(1): p. 1.

7. Maulina, T., et al., The Usage of Curcumin as Chemopreventive Agent for Oral Squamous Cell Carcinoma: An Experimental Study on Sprague-Dawley Rat. Integrative cancer therapies, 2019. 18: p. 1534735418822094.

8. Kewitz, S., I. Volkmer, and M.S. Staege, Curcuma contra cancer? Curcumin and Hodgkin's lymphoma. Cancer growth and metastasis, 2013. 6: p. 35. 
9. Cridge, B.J., L. Larsen, and R.J. Rosengren, Curcumin and its derivatives in breast cancer: Current developments and potential for the treatment of drug-resistant cancers. Oncology Discovery, 2013. 1(1): p. 6.

10. Reuter, S., et al., Modulation of anti-apoptotic and survival pathways by curcumin as a strategy to induce apoptosis in cancer cells. Biochemical pharmacology, 2008. 76(11): p. 1340-1351.

11. Salley, J.J., Experimental carcinogenesis in the cheek pouch of the Syrian hamster. Journal of dental research, 1954. 33(2): p. 253-262.

12. Sommariva, M., et al., High efficacy of CpG-ODN, Cetuximab and Cisplatin combination for very advanced ovarian xenograft tumors. Journal of translational medicine, 2013. 11(1): p. 1.

13. Manoharan, S., et al., Chemopreventive efficacy of curcumin and piperine during 7, 12-dimethylbenz (a) anthracene-induced hamster buccal pouch carcinogenesis. Singapore medical journal, 2009. 50(2): p. 139.

14. Lin, Y.-C., et al., Therapeutic efficacy evaluation of curcumin on human oral squamous cell carcinoma xenograft using multimodalities of molecular imaging. The American journal of Chinese medicine, 2010. 38(02): p. 343358.

15. Negi, A., et al., Comparison of Immunohistochemical Expression of Antiapoptotic Protein Survivin in Normal Oral Mucosa, Oral Leukoplakia, and Oral Squamous Cell Carcinoma. Pathology research international, 2015. 2015.

16. Casto, B.C., et al., Chemoprevention of oral cancer by lyophilized strawberries. Anticancer research, 2013. 33(11): p. 4757-4766.

17. Balakrishnan, S., et al., Effect of curcumin and ferulic acid on modulation of expression pattern of $\mathrm{p} 53$ and bcl-2 proteins in 7, 12-dimethylbenz () anthracene-induced hamster buccal pouch carcinogenesis. Indian J Biochem Biophys, 2010. 47: p 7-11.

18. Rajasekaran, D., et al., Proapoptotic, anti-cell proliferative, anti-inflammatory and antiangiogenic potential of carnosic acid during 7, 12 dimethylbenz (a) anthraceneinduced hamster buccal pouch carcinogenesis. African Journal of Traditional, Complementary and Alternative Medicines, 2013. 10(1): p. 102-112.

19. Arya, V., S. Singh, and M.J. Daniel, Clinicopathological correlation of $\mathrm{Bcl}-2$ oncoprotein expression in oral precancer and cancer. Journal of oral biology and craniofacial research, 2016. 6(1): p. 19-24.
20. Singh, B.B., et al., Immunohistochemical evaluation of bcl-2 oncoprotein in oral dysplasia and carcinoma. Oral Surgery, Oral Medicine, Oral Pathology, Oral Radiology, and Endodontology, 1998. 85(6): p. 692-698.

21. Afifi, M.M., et al., Therapeutic efficacy of plasmonic photothermal nanoparticles in hamster buccal pouch carcinoma. Oral surgery, oral medicine, oral pathology and oral radiology, 2013. 115(6): p. 743-751.

22. Sophia, J., Nimbolide, a neem limonoid inhibits Phosphatidyl Inositol-3 Kinase to activate Glycogen Synthase Kinase-3 $\beta$ in a hamster model of oral oncogenesis. Scientific reports, 2016. 6.

23. Manoharan, S., et al., Modulating effect of Enicostemma littorale on the expression pattern of apoptotic, cell proliferative, inflammatory and angiogenic markers During 7, 12-Dimethylbenz (a) anthracene induced hamster buccal pouch carcinogenesis. Toxicology international, 2015. 22(1): p. 130.

24. Manoharan, S., et al., Saffron reduction of 7, 12-dimethylbenz (a) anthracene-induced hamster buccal pouch carcinogenesis. Asian Pacific Journal of Cancer Prevention, 2013. 14(2): p. 951-957.

25. Nagini, S., et al., Of humans and hamsters: a comparative evaluation of carcinogen activation, DNA damage, cell proliferation, apoptosis, invasion, and angiogenesis in oral cancer patients and hamster buccal pouch carcinomas. Oral oncology, 2009. 45(6): p. e31-e37.

26. Rajasekaran, D., et al., Proapoptotic, anti-cell proliferative, anti-inflammatory and antiangiogenic potential of carnosic acid during 7, 12 dimethylbenz (a) anthraceneinduced hamster buccal pouch carcinogenesis. African Journal of Traditional, Complementary and Alternative Medicines, 2012. 10(1): p. 102-112.

27. Mohan, K.V.P.C., et al., Antiproliferative and apoptosis inducing effect of lactoferrin and black tea polyphenol combination on hamster buccal pouch carcinogenesis. Biochimica et Biophysica Acta (BBA)-General Subjects, 2006. 1760(10): p. 1536-1544.

28. Bisht, S. and A. Maitra, Systemic delivery of curcumin: 21st century solutions for an ancient conundrum. Current drug discovery technologies, 2009. 6(3): p. 192-199.

29. Kurzrock, R. and L. Li. Liposome-encapsulated curcumin: in vitro and in vivo effects on proliferation, apoptosis, signaling, and angiogenesis. in ASCO Annual Meeting Proceedings. 2005. 
30. Li, L., et al., Liposomal curcumin with and without oxaliplatin: effects on cell growth, apoptosis, and angiogenesis in colorectal cancer. Molecular cancer therapeutics, 2007. 6(4): p. 1276-1282.

31. Sandur, S.K., et al., Curcumin modulates the radiosensitivity of colorectal cancer cells by suppressing constitutive and inducible NF- $x \mathrm{~B}$ activity. International Journal of Radiation Oncology* Biology* Physics, 2009. 75(2): p. 534-542.
32. Hung, C.-M., et al., Demethoxycurcumin modulates prostate cancer cell proliferation via AMPK-induced downregulation of HSP70 and EGFR. Journal of agricultural and food chemistry, 2012. 60(34): p. 8427-8434.

33. Ravindran, J., S. Prasad, and B.B. Aggarwal, Curcumin and cancer cells: how many ways can curry kill tumor cells selectively? The AAPS journal, 2009. 11(3): p. 495-510.

34. Ismail, N.I., et al., Mechanism of apoptosis induced by curcumin in colorectal cancer. International journal of molecular sciences, 2019. 20(10): p. 2454. 